\title{
A Generic Optical Router Design for Photonic Network-on-Chips
}

\author{
Xianfang Tan, Student Member, IEEE, Mei Yang, Member, IEEE, Lei Zhang, Member, IEEE, \\ Yingtao Jiang, Member, IEEE, and Jianyi Yang, Member, IEEE
}

\begin{abstract}
Photonic network-on-chip (NoC) architectures are emerging as a new paradigm to interconnect a large number of processing cores at chip level, meeting the pressing demand for extremely high bandwidth and low power consumption. Optical routers, which are typically composed of silicon waveguides and optical switches, play a key role in an on-chip photonic interconnection network. In this paper, we propose a micro-ring-resonator (MRR)-based, scalable, and non-blocking passive optical router design, namely the generic wavelength-routed optical router (GWOR). We first introduce the four $4 \times 4$ GWOR router structures and then show how to construct GWORs of larger sizes by using the proposed $4 \times 4$ GWORs as the primitive building blocks. The number of MRRs used in the proposed GWOR is the least among the existing passive router designs for the same network size. In addition, we show that the power loss experienced on GWORs is lower than other comparative designs. Furthermore, to improve the bandwidth and fault tolerance capability of the GWORs, the redundant GWOR (RGWOR) structure is presented. RGWOR can provide multiple routing paths between each pair of input-output ports by cascading different types of GWORs.
\end{abstract}

Index Terms-Micro-ring resonator, photonic NoC, optical router.

\section{INTRODUCTION}

$\mathbf{T}$ HE rapid advance of technology continues to push up transistor integration capacity, which has enabled a large number of processing cores to be crapped into a chip multiprocessor (CMP) or a multiprocessor system-on-chip (MPSoC) design [8]. A vital challenge faced by many-core-based CMP and MPSoC designs is that their underline on-chip communication architectures must meet the large bandwidth and stringent latency requirements while not exceeding tight power budgets [1]. The continuously shrinking feature sizes, higher clock frequencies, and the simultaneous growth in complexity have made electronic network-on-chips (NoCs) a formidable task to provide scalable and power-efficient on-chip communication. Fortunately, the recent advances in nanoscale silicon

Manuscript received March 31, 2011; revised July 15, 2011; accepted October 19,2011. Date of publication December 07, 2011; date of current version January 27,2012 . This work was supported in part by the National Science Foundation under Grant CNS-1126688.

X. Tan, M. Yang, L. Zhang, and Y. Jiang are with the Department of Electrical and Computer Engineering, University of Nevada, Las Vegas, NV 89154 USA (e-mail: tanx@unlv.nevada.edu; Mei.Yang@unlv.edu; zhangl6@unlv.nevada. edu; Yingtao.Jiang@unlv.edu).

J. Yang is with the Department of Information Science and Electrical Engineering, Zhejiang University, Hangzhou, Zhejiang 310058, China (e-mail: yangjy@zju.edu.cn).

Color versions of one or more of the figures in this paper are available online at http://ieeexplore.ieee.org.

Digital Object Identifier 10.1109/JLT.2011.2178019

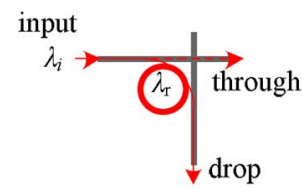

(a)

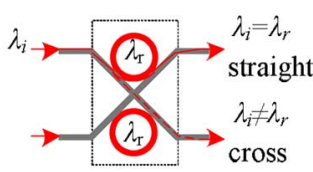

(b)
Fig. 1. (a) Basic operation of an MRR, and (b) a $2 \times 2$ optical switch.

photonics and development of silicon photonic devices, such as low-loss waveguides [10], [24], high-speed low-power-consumption modulators with up to $10 \mathrm{~Gb} / \mathrm{s}$ speed [11], [13], [25], hybrid-integrated evanescent lasers [5], [15], [18], and gigahertz-bandwidth SiGe photodetectors [1], [23], have made photonic NoCs a promising solution to meet the ever increasing chip-level interconnect challenges.

The heart of a photonic NoC is an on-chip photonic interconnection network which is composed of silicon waveguides and optical routers [17]. An optical router is generally built upon waveguides and optical switches, and as its name suggests, it optically routes data packets between a set of input and output ports. Of the many available optical switches, microring resonator (MRR)-based optical switches are typically preferred due to their ultra-compact size, simple-mode resonances, and ease of phase-matching between an MRR and its coupling waveguides [11]

The basic operation of an MRR is shown in Fig. 1(a). The input light signal is coupled to the drop port only if the input wavelength $\boldsymbol{\lambda}_{i}$ matches one of the resonance wavelengths of the MRR, say $\lambda_{r}$, which satisfies the following equation:

$$
m \lambda_{i}=n_{\mathrm{eff}} L
$$

where $m$ is an integer, $n_{\text {eff }}$ is the effective index of the optical mode, and $L$ is the length of the resonating cavity [17]. Otherwise, the input signal will simply pass to the through port.

By using MRRs of different sizes or tuning the refractive index through either thermo-optic (TO) [21] or electro-optic (EO) effect [17], an incoming optical signal can be switched to the destined output port solely based on the signal wavelength. Fig. 1(b) shows a $2 \times 2$ optical switch constructed by two identical MRRs.

The performance of on-chip photonic interconnection designs can be evaluated in terms of the bandwidth, power consumption, footprint, and scalability. Existing photonic NoC designs can be classified into two classes [17]: 1) switching networks using TO or EO effect tuning switches, and 2) passive networks using switches with a fixed-wavelength assignment. 
Switching networks [20], [21] in general provide higher bandwidths, but they require high-speed electrical control circuits be integrated with the photonic interconnections, which tends to be very difficult to realize and will have adverse impacts on power consumption. Passive networks, on the other hand, can route data with fixed wavelength and do not require extra control circuits; as so, passive networks tend to have better power performance. However, all the existing passive networks [3], [9] suffer from poor scalability and high design complexity, making active switching networks remain the preferred choice of design for large scale optical NoC systems [14], [20].

In this paper, we focus on passive networks and propose the generic wavelength-routed optical router (GWOR) to address the scalability problems inherent in passive photonic NoCs. We first introduce the $4 \times 4$ GWOR router structure and then show how to construct GWORs of larger sizes using the proposed $4 \times 4$ GWOR as the basic building block. The wavelength assignment schemes for GWOR of any size are also derived. Comparisons of GWOR with several existing routers confirm that the proposed GWOR uses the least number of MRRs and has the lowest power loss among all the optical routers of the same size. More importantly, the proposed GWOR is proved to be non-blocking, which is a desirable property for optical routers.

The rest of this paper is organized as follows. Section II briefly reviews the related work on on-chip optical routers. Section III presents the structure of $4 \times 4$ GWOR and Section IV shows how to construct GWORs of any larger sizes using the $4 \times 4$ GWOR as the basic building block. In Section V, we compare GWORs with existing optical routers in terms of the number of MRRs required and the optical power loss. In Section VI, a redundant GWOR structure is proposed to improve the bandwidth and fault tolerance capability. Finally, Section VII concludes the paper.

\section{RELATED WORK}

In recent years, a number of MRR-based on-chip optical routers have been proposed and they can be categorized based on their topologies [17]: 1) Cross-grid type and 2) Banyan network type. Optical routers can also be categorized based on the signal-switching mode in the router: 1) active routers where data switching is realized by tuning MRRs, and 2) passive routers where routing is based on MRRs each with a fixed-routing wavelength.

In existing on-chip optical active routers, the first fabricated router is a TO-tuned glass MRR-based $8 \times 8$ optical crossbar presented in [12] for the sole purpose of experimental demonstration. In [16], a $5 \times 5$ EO-tuned silicon photonic crossbar is reported and this improved crossbar eliminates the self-routing as the case in the traditional crossbar structures in [17]. An optimized $5 \times 5$ TO-tuned optical crossbar called Cygnus router is proposed in [6], which aligns the ports to the corresponding directions as required in regular $\mathrm{NoC}$ topologies (e.g., mesh/torus). In Cygnus router, four waveguides are bent and the internal structure of the traditional crossbar is reoriented to reduce the number of waveguide crossings. However, the power loss on each routing path may be elevated due to more waveguide bending. A $4 \times 4$ TO-tuned bidirectional hitless router is fabricated and reported in [21]. However, it is not clear how this router can be scaled up to build routers larger than $4 \times 4$, and this scalability problem imposes a serious restriction when designing an on-chip mesh/torus network. All the active routers listed above fall into cross-grid type and they are non-blocking. In general, these active cross-grid routers are compact in size, but their scalability is limited by the additional insertion loss and crosstalk at the waveguide crossings. Furthermore, as the data routing in active routers is realized by tuning MRRs, extra control circuitry is required for tuning each MRR by either TO or EO effect. In terms of effectiveness, TO-based tuning can achieve a wavelength tuning range of $20 \mathrm{~nm}$ [11], at a cost of extra heating/cooling time (in microseconds) and higher power consumption $(0.25 \mathrm{~nm} / \mathrm{mW})$ [21]. On the other hand, the EO-based tuning currently can only reach wavelength tuning range of $2 \mathrm{~nm}$ [21] with driving power ranging from 18 to $105 \mu \mathrm{W}$ [20].

As for passive routers, wavelength-based routing is used and the routing patterns are determined at design time. Being the first passive router ever proposed for NoC, the $\lambda$-router [3] is constructed by cascading MRR-based switches to form a Banyan network. But this router architecture is only applicable to networks with even-numbered input/output ports. This parity problem is later solved by a more general WRON router [26], [27]. Another passive optical router is the oblivious bidirectional $5 \times 5$ cross-grid wavelength-router presented in [9], which is compact in size, but the high design complexity makes it difficult to be extended to construct a larger sized network. In [8], a U-shape passive optical router is introduced which in fact implements a wavelength-routed crossbar structure. The passive optical routers listed above are non-blocking. In [28], another cascaded $N \times N$ wavelength-routed network is proposed, which, unfortunately, is an incomplete crossbar as not all input signals can be routed to any of the outputs (e.g., for the given $3 \times 3$ router, there is no routing path between $I_{2}$ and $O_{3}$ ). While in [14], a $4 \times 4$ 64-wavelength optical crossbar is proposed in which each port can route 16 different wavelengths. However, this design is actually blocking. For instance, simultaneous data transmission from $I_{1} \rightarrow O_{0}$ and $I_{3} \rightarrow O_{1}$ will cause a routing confliction.

To address the problems existing in the aforementioned work, in this paper, we propose GWOR, a generic wavelength-routed, bidirectional optical router that is non-blocking, scalable, and compact in size.

\section{ROUTER ARCHITECTURE OF $4 \times 4$ GWOR}

In this section, the $4 \times 4$ GWOR is first introduced as the primitive building blocks to build GWORs of larger sizes. As shown in Fig. 2, a $4 \times 4$ GWOR consists of two horizontal and two vertical waveguides. For each waveguide, it has one and only one intersection with each one of the waveguides on the orthogonal direction. As so, the four waveguides together form a primitive checkerboard-shaped cell. The following labeling conventions are followed when labeling the ports of the $4 \times 4$ GWOR.:

1) The two ports located at each of the four directions (i.e., north $(N)$, west $(W)$, south $(S)$, and east $(E)$ ) of the primitive checkerboard-shaped cell, are grouped and labeled as 


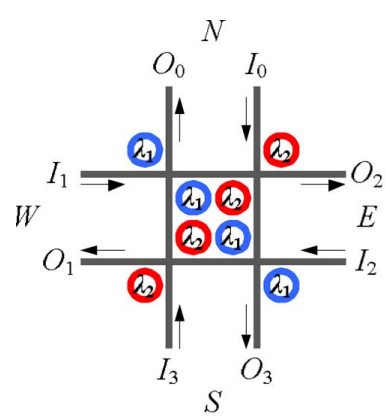

(a)

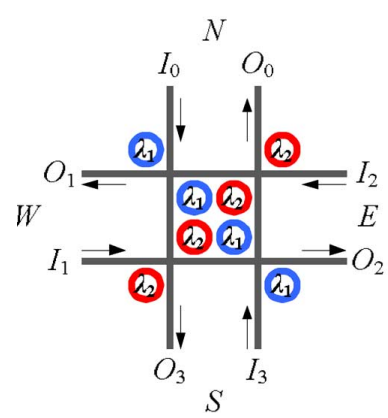

(c)

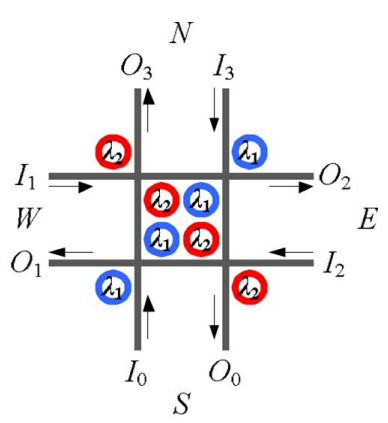

(b)

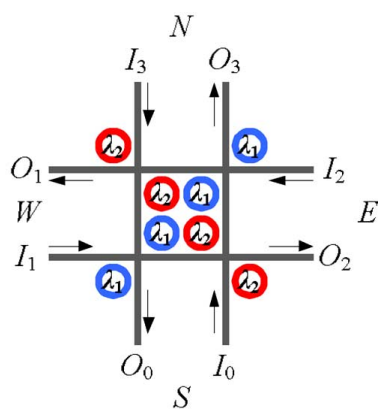

(d)
Fig. 2. Basic $4 \times 4$ GWOR structures. (a) Type I. (b) Type II. (c) Type III. (d) Type IV.

one birdirectional port such as $P_{i}\left(I_{i}, O_{i}\right)$, where $I(O)$ represents an input (output) port. In one $4 \times 4$ GWOR, the input and output are labeled in the same order for each bidirectional port, either clockwise or counterclockwise.

2) Each waveguide is dedicated for the direct connection between an input-output pair, denoted as $I_{i} \rightarrow O_{3-i}(i=$ $0,1,2,3)$.

Following the labeling convention above, one can see that a total of four types of $4 \times 4$ GWOR can be formed. Fig. 2(a) shows the structure of Type I $4 \times 4$ GWOR. The four ports are labeled, starting from the north side and traversing in counterclockwise direction, as $P_{0}\left(I_{0}, O_{0}\right)$ to $P_{1}\left(I_{1}, O_{1}\right)$. As a consequence of direct connections of ports, the ports at the south and west sides are labeled as $P_{3}\left(I_{3}, O_{3}\right)$ and $P_{2}\left(I_{2}, O_{2}\right)$, respectively. At each direction, the input and output ports are always ordered in counterclockwise manner.

Figs. 2(b)-(d) show the structures of the Type II, III and IV $4 \times 4$ GWORs, respectively. The distinction between the Type I and II $4 \times 4$ GWORs is that in Type II GWOR, the ports are numbered starting from the south side as $P_{0}\left(I_{0}, O_{0}\right), P_{1}$ $\left(I_{1}, O_{1}\right), P_{3}\left(I_{3}, O_{3}\right)$, and $P_{2}\left(I_{2}, O_{2}\right)$ in clockwise manner. The Type III $4 \times 4$ GWOR has the same order of ports in directions as the Type I $4 \times 4$ GWOR but differs from Type I in that the input and output ports at each direction are ordered in clockwise manner instead. Similarly, Type IV $4 \times 4$ GWOR has the same order of ports in directions as the Type II $4 \times 4$ GWOR but differs from Type II in that the input and output ports at each direction are ordered in clockwise manner instead.

For GWORs, non-blocking routing is realized by assigning MRRs to the appropriate corners of the intersections of waveguides so that all the input light signals can be directed to their
TABLE I

ROUTING WAVELENGTH AssignMENT OF THE $4 \times 4$ GWOR

\begin{tabular}{|c|c|c|c|c|}
\hline & $O_{0}$ & $O_{1}$ & $O_{2}$ & $O_{3}$ \\
\hline$I_{0}$ & - & $\lambda_{1}$ & $\lambda_{2}$ & $\lambda_{3}$ \\
\hline$I_{1}$ & $\lambda_{1}$ & - & $\lambda_{3}$ & $\lambda_{2}$ \\
\hline$I_{2}$ & $\lambda_{2}$ & $\lambda_{3}$ & - & $\lambda_{1}$ \\
\hline$I_{3}$ & $\lambda_{3}$ & $\lambda_{2}$ & $\lambda_{1}$ & - \\
\hline
\end{tabular}

designated output ports. Assuming no self-communication is allowed (i.e., input signal from $I_{i}$ will not go to $O_{i}$ ), there exist 12 possible input-output pairs in a $4 \times 4$ GWOR. As four pairs $\left(I_{i} \rightarrow O_{3-i}\right)$ are directly connected by the four waveguides, at least eight MRRs are needed to realize the routing for the remaining eight pairs.

To implement wavelength-based routing, MRRs should be assigned with different sizes (or resonant wavelengths). The principle of wavelength assignment is to assign the minimal number of wavelengths to the input-output pairs so that any input-output communication can be realized without causing a confliction. Given the input-output pair $\left(I_{i} \rightarrow O_{j}\right)$, the input wavelength $C(i, j)$ is determined by

$$
C(i, j)= \begin{cases}- & i=j \\ \lambda_{3} & i+j=3, i \neq j \\ \lambda_{\bmod (2 j, 3)} & i=3,0<j<3 \\ \lambda_{\bmod (3-2 i, 3)} & j=0,0<i<3 \\ \lambda_{\bmod (j-i, 3)} & \text { others. }\end{cases}
$$

Table I lists the wavelength assigned to each input-output pair of $4 \times 4$ GWORs. To minimize the number of wavelengths, it can be seen from Table I that whenever possible, wavelengths are shared, i.e., $C(i, j)=C(3-i, 3-j)$. It shall be noted that the same set of wavelengths will cover all the rows and the columns in Table I.

According to the wavelength assignment in Table I, to route the light signals from $I_{i}$ to $O_{j}$ and from $I_{3-i}$ to $O_{3-j}(i, j=$ $0,1,2$ or $3, i \neq j$, and $i+j \neq 3$ ), two identical MRRs corresponding to the assigned input wavelength are placed at the corners of the intersection where the light signals shall make turn to reach their designated output ports. As shown in Fig. 2, only two types of MRRs with a total of 8 MRRs are needed for each type of the $4 \times 4$ GWORs.

The four types of $4 \times 4$ GWORs shown in Fig. 2 are isomorphic in terms of routing since they share the same routing wavelength assignment. It is easy to verify that the $4 \times 4$ GWOR is non-blocking for any unicast communication using the input wavelength assignment as tabulated in Table I. In addition, multicasting and broadcasting can also be supported by simply multiplexing multiple input wavelengths at any input port.

\section{Generalization of GWOR}

\section{A. Generalization of GWOR to Even-Numbered Inputs/Outputs}

The four types of $4 \times 4$ GWORs introduced in Section III are used as the primitive building blocks to create larger sized GWORs. Correspondingly, four types of $N \times N$ GWORs (where $N>4$ ) can be built based on each type of $4 \times 4$ GWOR. In this section, we will consider the even-numbered $N \times N$ GWORs (where $N=2 n$ and $n>2$ ). In an $N \times N$ GWOR, $N$ 


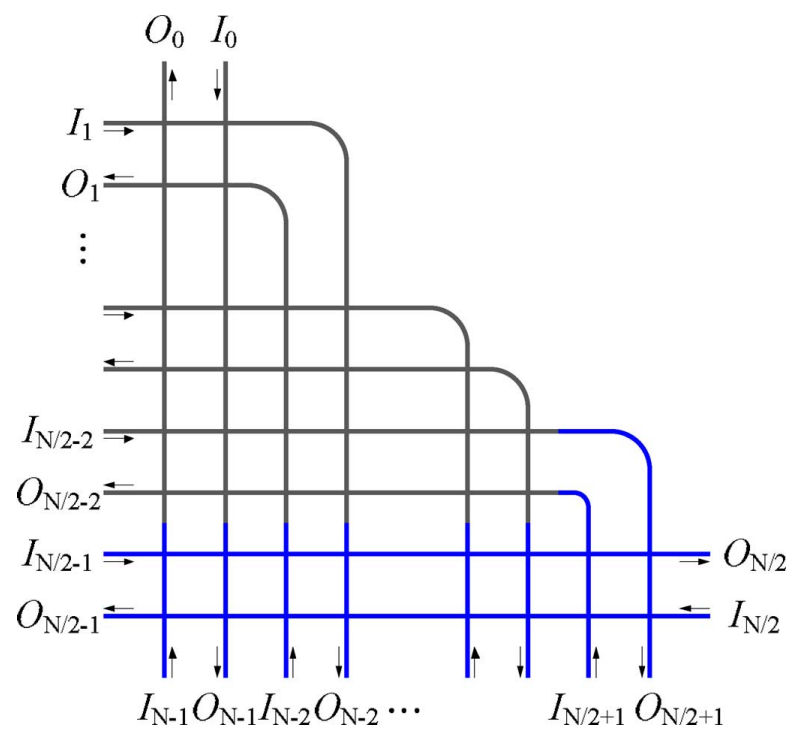

Fig. 3. Type I $\mathrm{N} \times \mathrm{N}$ GWOR built on Type I $(N-2) \times(N-2)$ GWOR.

waveguides are used and each waveguide is dedicated for the direct connection for one pair of input-output ports, denoted as $I_{i} \rightarrow O_{j}$ (where $i, j=0,1, \ldots, N-1$, and $i+j=N-1$ ). The $N$ waveguides are partitioned into $N / 2$ groups and each group consists of two parallel waveguides. Taking the type I $N \times N$ GWOR as an example, the following rules are followed to interconnect the $N / 2$ groups of waveguides.

1) The first two groups of waveguides are laid out as in the $4 \times 4$ GWOR (Fig. 2(a)). The vertical group consists of two parallel waveguides $I_{0} \rightarrow O_{N-1}$ and $I_{N-1} \rightarrow O_{0}$, while the horizontal group consists of $I_{1} \rightarrow O_{N-2}$ and $I_{N-2} \rightarrow$ $O_{1}$. The intersection of the two waveguide groups forms a primitive $4 \times 4$ GWOR. Assume the waveguides in $4 \times 4$ GWOR have equal length of $l$ and let $i=2$.

2) Extend all the waveguides of the router created so far by $2 l / 3$. For the horizontal waveguide group, bend the east end of each waveguide so that it continues to go south and keep the two bent waveguides in parallel. Then the bent waveguides are extended until they align themselves with the vertical waveguides.

3) Add another group of waveguides horizontally to the extended router after completing Step 2, and have the newly added waveguide group intersect with all the existing waveguide groups. The newly added group waveguides are labeled as $I_{i} \rightarrow O_{N-1-i}$ and $I_{N-1-i} \rightarrow O_{i}$.

4) If there are more waveguide groups to be added, let $i=$ $i+1$, and add them one at a time following Steps 2 and 3.

Fig. 3 shows how Type I $N \times N$ GWOR is constructed from Type I $(N-2) \times(N-2)$ GWOR, where both the extended sections of vertical waveguides and the newly added waveguides are colored in blue. One can see that for any two groups of waveguides, their intersections form one and only one primitive $4 \times 4$ GWOR. Hence, there is one and only one intersection between any two orthogonal waveguides. The $N$ ports are labeled, from the top ends of the vertical waveguides in counterclockwise manner, as $P_{0}\left(I_{0}, O_{0}\right), P_{1}\left(I_{1}, O_{1}\right), \ldots, P_{N / 2-1}\left(I_{N / 2-1}, O_{N / 2-1}\right)$,
TABLE II

WAVELENGTH ASSIGNMENT OF $8 \times 8$ GWOR

\begin{tabular}{|c|c|c|c|c|c|c|c|c|}
\hline & $O_{0}$ & $O_{1}$ & $O_{2}$ & $O_{3}$ & $O_{4}$ & $O_{5}$ & $O_{6}$ & $O_{7}$ \\
\hline$I_{0}$ & - & $\lambda_{1}$ & $\lambda_{2}$ & $\lambda_{3}$ & $\lambda_{4}$ & $\lambda_{5}$ & $\lambda_{6}$ & $\lambda_{7}$ \\
\hline$I_{1}$ & $\lambda_{5}$ & - & $\lambda_{1}$ & $\lambda_{2}$ & $\lambda_{3}$ & $\lambda_{4}$ & $\lambda_{7}$ & $\lambda_{6}$ \\
\hline$I_{2}$ & $\lambda_{3}$ & $\lambda_{6}$ & - & $\lambda_{1}$ & $\lambda_{2}$ & $\lambda_{7}$ & $\lambda_{4}$ & $\lambda_{5}$ \\
\hline$I_{3}$ & $\lambda_{1}$ & $\lambda_{5}$ & $\lambda_{6}$ & - & $\lambda_{7}$ & $\lambda_{2}$ & $\lambda_{3}$ & $\lambda_{4}$ \\
\hline$I_{4}$ & $\lambda_{6}$ & $\lambda_{4}$ & $\lambda_{5}$ & $\lambda_{7}$ & - & $\lambda_{1}$ & $\lambda_{2}$ & $\lambda_{3}$ \\
\hline$I_{5}$ & $\lambda_{4}$ & $\lambda_{3}$ & $\lambda_{7}$ & $\lambda_{5}$ & $\lambda_{6}$ & - & $\lambda_{1}$ & $\lambda_{2}$ \\
\hline$I_{6}$ & $\lambda_{2}$ & $\lambda_{7}$ & $\lambda_{3}$ & $\lambda_{4}$ & $\lambda_{5}$ & $\lambda_{6}$ & - & $\lambda_{1}$ \\
\hline$I_{7}$ & $\lambda_{7}$ & $\lambda_{2}$ & $\lambda_{4}$ & $\lambda_{6}$ & $\lambda_{1}$ & $\lambda_{3}$ & $\lambda_{5}$ & - \\
\hline
\end{tabular}

and their corresponding directly-connected ports are $P_{N-1}\left(I_{N-1}, O_{N-1}\right), P_{N-2}\left(I_{N-2}, O_{N-2}\right), \ldots, \quad P_{N / 2}\left(I_{N / 2}\right.$, $\left.O_{N / 2}\right)$. Similar to the Type I $4 \times 4$ GWOR, in Type I $N \times N$ GWOR, the input and output of each bidirectional port are labeled in counterclockwise direction.

To build a Type II $N \times N$ GWOR, similar rules to one used in the construction of Type I $N \times N$ GWOR are applied except that: 1) in Step 1, Type II $4 \times 4$ GWOR [Fig. 2(b)] is used, 2) in Step 2, the vertical waveguides are extended to the north and the horizontal waveguides are bent to the north direction. Note that the $N$ ports are labeled from the bottom ends in clockwise manner as $P_{0}\left(I_{0}, O_{0}\right), \quad P_{1}\left(I_{1}, O_{1}\right), \ldots, \quad P_{N / 2-1}\left(I_{N / 2-1}, O_{N / 2-1}\right)$ and their corresponding directly-connected ports from $P_{N-1}\left(I_{N-1}, O_{N-1}\right), \quad P_{N-2}\left(I_{N-2}, O_{N-2}\right), \ldots, P_{N / 2}\left(I_{N / 2}\right.$, $\left.O_{N / 2}\right)$.

The type III and IV $N \times N$ GWORs can be built following the rules similar to the ones used when constructing Types I and II $N \times N$ GWORs, except that in Step 1 , Type III $4 \times 4$ GWOR is used for Type III $N \times N$ GWOR while Type IV $4 \times 4$ GWOR for Type IV $N \times N$ GWOR.

Similar to that of $4 \times 4$ GWORs, routing of $N \times N$ GWORs is realized by placing MRRs to the appropriate corners of the waveguide intersections based on the wavelength assignment scheme derived below. Given the input-output pair $I_{i} \rightarrow O_{j}$, the input wavelength $C(i, j)$ is determined by

$$
\begin{aligned}
& C(i, j) \\
& = \begin{cases}- & i=j \\
\lambda_{N-1} & i+j=N-1, i \neq j \\
\lambda_{\bmod (2 j, N-1)} & i=N-1,0<j<N-1 \\
\lambda_{\bmod (N-1-2 i, N-1)} & j=0,0<i<N-1 \\
\lambda_{\bmod (j-i, N-1)} & \text { others. }\end{cases}
\end{aligned}
$$

Table II tabulates the wavelength assigned to each input-output pair of $8 \times 8 \mathrm{GWORs}$. To route light signals from $I_{i}$ to $O_{j}(i, j=0,1, \ldots, N-1)$, an MRR with the assigned wavelength is placed at the intersection of the waveguides connecting $I_{i}$ and $O_{j}$ where the values of $i$ and $j$ have to satisfy the conditions of $i \neq j$ and $i+j \neq N-1$. It can be seen from Table II that the input-output pairs with direct connections are assigned with the same wavelength (e.g., $\lambda_{7}$ in the $8 \times 8$ GWOR), which ensures that minimum number of different types of MRRs is used in a GWOR. Figs. 4(a) and (b) show the constructed Type I and II $8 \times 8$ GWOR structure, respectively, where only 6 types of MRRs are needed for each type.

Type III $8 \times 8$ GWOR differs from its Type I counterpart only in its labeling order of the input and output for each bidirectional port since both types follow the same wavelength assignment. 


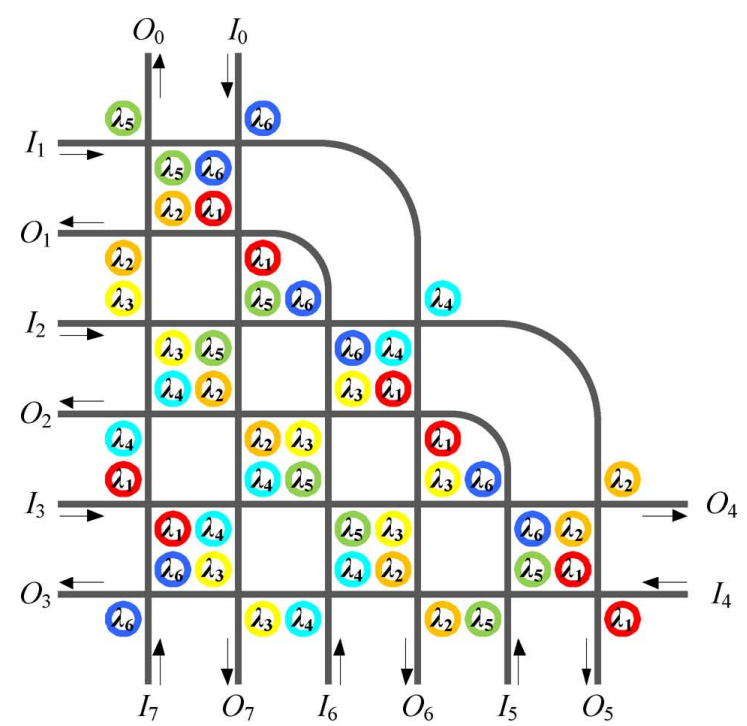

(a)

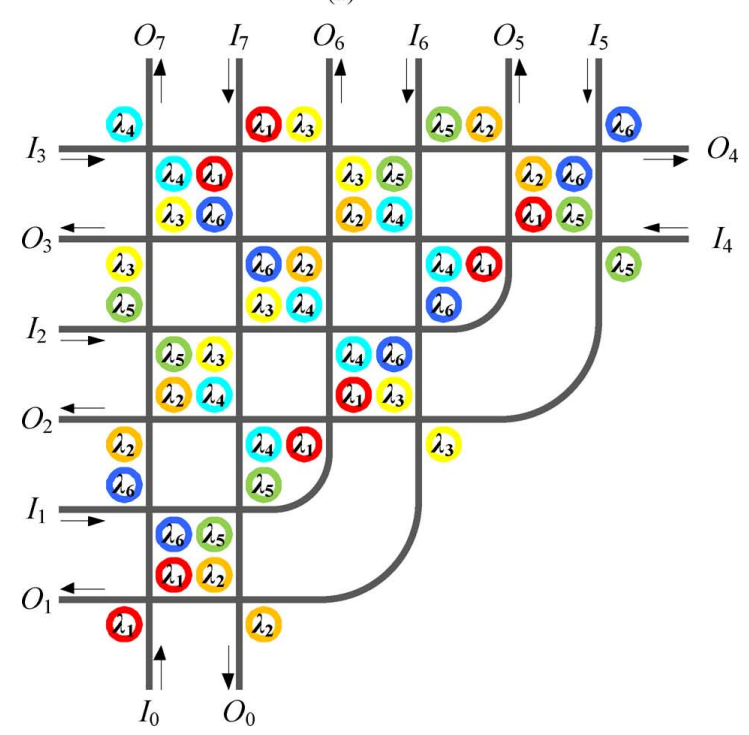

(b)

Fig. 4. Structure of $8 \times 8$ GWORs. (a) Type I $8 \times 8$ GWOR. (b) Type II $8 \times 8$ GWOR.

Similarly, Type IV GWOR differs from its Type II counterpart in the labeling order of the input and output for each bidirectional port. Both Types II and IV $8 \times 8$ GWORs follow the same wavelength assignment as their Types I and III counterparts. As such, Types I, II, III and IV $N \times N$ GWORs are isomorphic in terms of routing.

The properties of an $N \times N$ GWOR $(N=2 n$ and $n \geq 2)$ are given below.

Lemma 1: For an $N \times N$ GWOR, the intersections of $N / 2$ waveguide groups form $N(N-2) / 8$ primitive checkerboardshaped cell.

Proof: As shown in the construction rules of $N \times N$ GWOR, every two groups form a primitive checkerboard-shaped cell as in Fig. 2. Hence the lemma holds.

Proposition 1: The total number of MRRs in an $N \times N$ GWOR is $N(N-2)$, which is the minimum number for an $N \times N$ router.
Proof: As in Section III, 8 MRRs are assigned to each primitive checkerboard-shaped cell. Thus, based on Lemma 1, totally $N(N-2) / 8 \times 8=N(N-2)$ MRRs will be used.

As no self-communication is allowed in $N \times N$ GWORs (i.e., no input signal from $I_{i}$ will go to $\left.O_{i}\right)$, there exist $N(N-1)$ possible input-output pairs in an $N \times N$ GWOR. As $N$ pairs of $\left(I_{i} \rightarrow O_{N-i}\right)$ are directly connected by $N$ waveguides, at least $N(N-1)-N=N(N-2)$ MRRs are needed to realize the routing of the rest $N(N-2)$ pairs. Hence the proposition holds.

Lemma 2: The number of different MRR types for an $N \times$ $N$ GWOR is $N-2$, and their corresponding wavelengths are $\lambda_{1}, \lambda_{2}, \ldots, \lambda_{N-2}$.

Proof: From (3), $N-1$ input wavelengths are needed. Hence $N-2$ different types of MRRs are needed since no MRR is used for a directly connected input-output pair.

Proposition 2: The $N \times N$ GWORs are non-blocking.

Proof: According to the rules of constructing $N \times N$ GWORs, for any type of $N \times N$ GWOR, each waveguide has one and only one intersection with each waveguide in the other $(N-2) / 2$ groups. Therefore, there are totally $(N-2)$ intersections between one waveguide and the other waveguides. Equation (3) ensures that for each input, a distinct input wavelength is assigned for a different output. Accordingly, at the intersection of the waveguides connected to $I_{i}$ and $O_{j}(i \neq j, i+j \neq N-1)$, the MRR corresponding to the input wavelength of $I_{i} \rightarrow O_{j}$ is assigned. As a result, along each waveguide, $N-2$ different types of MRRs are assigned and two identical MRRs only appear at the diagonal corner of a waveguide intersection.

Consequently, on the $N \times N$ GWOR, for input-output pair $I_{i} \rightarrow O_{j}(i \neq j, i+j \neq N-1)$, the light signal from $I_{i}$ with the assigned wavelength (governed by (3)) will be dropped to $O_{j}$ at the exact intersection with the corresponding MRR. For directly connected input-output pair $I_{i} \rightarrow O_{j}(i \neq j, i+j=N-1)$, the input signal with the assigned wavelength will simply go straight to reach $O_{j}$. That is, all the $N-1$ output ports can be reached from any input port. In addition, inside any one waveguide, the light signals travelling between different input-output pairs through the same waveguide will not interfere each other because they use different wavelengths. Hence the proposition holds.

\section{B. Generalization of GWOR to Odd-Numbered Inputs/Outputs}

The method introduced for even-numbered GWORs in previous subsection can be adapted to build $N \times N$ GWORs, where $N=2 n+1$ and $n \geq 2$. Here, the $N$ waveguides are divided into $(n+1)$ groups, among which the first $n$ groups are the same as the even number cases, but the $(n+1)_{t h}$ group contains only one waveguide $\left(I_{n} \rightarrow O_{n}\right)$. The construction procedure introduced in Section IV. $A$ is followed to interconnect the $N$ waveguides. The only difference is that at the last step, each intersection formed by the $(n+1)_{t h}$ group with any other waveguide group will only contain the two upper crossings of a primitive checkerboard-shaped cell, namely a reduced primitive checkerboard-shaped cell. Obviously, four types of $N \times N$ GWORs can 
TABLE III

WAVELENGTH ASSIGNMENT OF MRR-BASED $5 \times 5$ GWOR ROUTER

\begin{tabular}{|c|c|c|c|c|c|}
\hline & $O_{0}$ & $O_{1}$ & $O_{2}$ & $O_{3}$ & $O_{4}$ \\
\hline$I_{0}$ & - & $\lambda_{1}$ & $\lambda_{2}$ & $\lambda_{3}$ & $\lambda_{4}$ \\
\hline$I_{1}$ & $\lambda_{4}$ & - & $\lambda_{1}$ & $\lambda_{2}$ & $\lambda_{3}$ \\
\hline$I_{2}$ & $\lambda_{3}$ & $\lambda_{4}$ & - & $\lambda_{1}$ & $\lambda_{2}$ \\
\hline$I_{3}$ & $\lambda_{2}$ & $\lambda_{3}$ & $\lambda_{4}$ & - & $\lambda_{1}$ \\
\hline$I_{4}$ & $\lambda_{1}$ & $\lambda_{2}$ & $\lambda_{3}$ & $\lambda_{4}$ & - \\
\hline
\end{tabular}

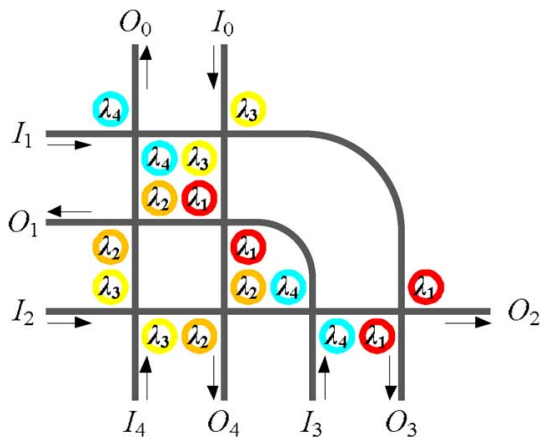

Fig. 5. Structure of Type I $5 \times 5$ GWOR

be built based on the four types of $4 \times 4$ GWORs. For odd-numbered GWORs, given the input-output pair $I_{i} \rightarrow O_{j}$, the input wavelength assignment $C(i, j)$ is determined by

$$
C(i, j)= \begin{cases}- & i=j \\ \lambda_{\bmod (j-i, N)} & \text { others. }\end{cases}
$$

Table III shows the wavelength assignment of $5 \times 5$ GWOR. An MRR with assigned wavelength is placed at the waveguide intersections to direct light signals from $I_{i}$ to $O_{j}$ when $i$ and $j$ satisfy $i \neq j$ and $i+j \neq N-1$. Based on the wavelength assignment tabulated in Table III, a $5 \times 5$ GWOR is shown in Fig. 5.

The properties of an $N \times N$ GWOR (where, $N=2 n+1$ and $n \geq 2$ ) are summarized below.

Lemma 3: For an $N \times N$ GWOR, the intersections of the $(n+1)$ groups of the waveguides form $n(n-1) / 2$ primitive checkerboard-shaped cells and $n$ reduced primitive checkerboard-shaped cells.

Proof: As shown in the construction rules of $N \times N$ GWOR, according to Lemma 1, the intersections of the first $n$ group of waveguides form $C_{n}^{2}=C_{(N-1) / 2}^{2}=n(n-1) / 2$ primitive checkerboard-shaped cells and the intersections of the $(n+1)_{t h}$ group with the first $n$ groups form $n$ reduced primitive checkerboard-shaped cells.

Proposition 3: The total number of MRRs used in an $N \times N$ GWOR $(N=2 n+1)$ is $(N-1)^{2}$, and this is the minimum number for an $N \times N$ router.

Proof: As in Section III, 8 MRRs are assigned to each primitive checkerboard-shaped cell and 4 MRRs are assigned to each reduced primitive checkerboard-shaped cell. Based on Lemma 3, totally $n(n-2) / 2 \times 8+n \times 4=4 n^{2}=(N-1)^{2}$ MRRs will be used.

Similar to the proof of Proposition 1, as no self-communication is allowed in the $N \times N$ GWOR and there exist $N(N-1)$ possible input-output pairs in it, among which there are $(N-$ 1) pairs $\left(I_{i} \rightarrow O_{N-i}\right)$ are directly connected by waveguides, hence, at least $N(N-1)-(N-1)=(N-1)^{2}$ MRRs are
TABLE IV

Number of MRRS USED IN DIFFERENT SIZE ROUTERS

\begin{tabular}{|c|c|c|c|c|c|c|}
\hline MRRs & Crossbar & Reduced Crossbar & Hitless Router & WRON & $\lambda$-router & GWOR \\
\hline $4 \times 4$ & 16 & 12 & 8 & 12 & 12 & 8 \\
\hline $5 \times 5$ & 25 & 20 & - & 20 & - & 16 \\
\hline $6 \times 6$ & 36 & 30 & - & 30 & 30 & 24 \\
\hline $7 \times 7$ & 49 & 42 & - & 42 & - & 36 \\
\hline $8 \times 8$ & 64 & 56 & - & 56 & 56 & 48 \\
\hline
\end{tabular}

needed to realize the routing of the rest $(N-1)^{2}$ indirectly-connected pairs. Hence the proposition holds.

Lemma 4: The total number of different types of MRRs for a $N \times N$ GWOR is $N-1$, and their corresponding wavelengths are $\lambda_{1}, \ldots, \lambda_{N-1}$.

Proof: Based on (4), $N-1$ input wavelengths are needed as for odd number cases. However, different from even number cases, $N-1$ different types of MRRs are needed on the intersections formed by the waveguide $\left(I_{n} \rightarrow O_{n}\right)$ with other waveguides.

Proposition 4: The constructed $N \times N$ GWOR is non-blocking.

Proof: The Proof is similar to that for Proposition 2.

\section{COMPARISON AND ANALYSIS}

In this section, we compare the number of MRRs used and estimated optical power loss in GWORs against several existing typical router designs, including the matrix-based crossbar [16], the reduced crossbar [17], the hitless router [21], the WRON [26], [27], and the $\lambda$-router [3].

The number of MRRs used in an $N \times N$ crossbar is $N^{2}$, which can be reduced to $N(N-1)$ in a reduced crossbar, WRON or $\lambda$-router (with even-numbered sizes only). For an $N \times N$ GWOR, the number of MRRs used is $N(N-2)$ (as Proposition 1 for $N=2 n$ and $n \geq 2$ ) or $(N-1)^{2}$ (as Proposition 3 for $N=2 n+1$ and $n \geq 2$ ). Table IV shows the number of MRRs used in GWORs and other routers of the same sizes. It can be seen that GWOR uses the least number of MRRs among all the routers. An exceptional case is that for $4 \times 4$ routers, both hitless router and GWOR use the smallest number of MRRs ( 8 MRRs).

To evaluate the optical power loss experienced in all the routers listed in Table IV, the power loss parameters given in [2] are adopted here: each MRR has a drop-loss of $1.5 \mathrm{~dB}$ and a through-loss of $0.01 \mathrm{~dB}$, and the crossing loss and bending loss of waveguides are $0.05 \mathrm{~dB}$ and $0.013 \mathrm{~dB}$, respectively. Therefore, for a given input-output pair $\left(I_{i} \rightarrow O_{j}\right.$, where $i, j=0,1, \ldots, N-1$, and $i \neq j$ ), the total power loss on the routing path can be estimated by

$$
\begin{aligned}
P_{\text {loss }}(i, j)=1.5 \times & N_{\text {drop }}+0.01 \times N_{\text {through }} \\
& +0.05 \times N_{\text {crossing }}+0.013 \times N_{\text {bending }}
\end{aligned}
$$

where 1) $N_{\text {drop }}$ denotes the number of drop-losses, and it is determined by the number of resonances made on the routing path, 2) $N_{\text {through }}$ denotes the number of through-losses, and it is determined by the number of MRRs passed by a light signal, 3) $N_{\text {crossing }}$ denotes the number of waveguide crossings, and 4) $N_{\text {bending }}$ is the number of waveguide bendings. The average power loss is the arithmetic mean of all possible $P_{\text {loss }}(i, j)$ in the given router. 
TABLE V

POWER Loss Estimation OF DifFERENT SizE Routers

\begin{tabular}{|c|c|c|c|c|c|c|c|c|c|c|c|c|}
\hline \multirow{2}{*}{$\begin{array}{c}\text { Power } \\
\text { Loss }(d B)\end{array}$} & \multicolumn{2}{|c|}{ Crossbar } & \multicolumn{2}{|c|}{$\begin{array}{c}\text { Red. } \\
\text { Crossbar }\end{array}$} & \multicolumn{2}{|c|}{$\begin{array}{l}\text { Hitless } \\
\text { Router }\end{array}$} & \multicolumn{2}{|c|}{ WRON } & \multicolumn{2}{|c|}{$\lambda$-router } & \multicolumn{2}{|c|}{ GWOR } \\
\hline & Max & Avg & Max & Avg & Max & Avg & Max & Avg & Max & Avg & Max & Avg \\
\hline $4 \times 4$ & 1.86 & 1.68 & 1.84 & 1.67 & 1.73 & 1.16 & 1.71 & 1.29 & 1.71 & 1.30 & 1.64 & 1.09 \\
\hline $5 \times 5$ & 1.98 & 1.74 & 1.98 & 1.73 & - & - & 1.78 & 1.43 & - & - & 1.79 & 1.37 \\
\hline $6 \times 6$ & 2.10 & 1.80 & 2.10 & 1.79 & - & - & 1.85 & 1.55 & 1.85 & 1.55 & 1.93 & 1.40 \\
\hline $7 \times 7$ & 2.32 & 1.86 & 3.32 & 1.85 & - & - & 1.92 & 1.71 & - & - & 2.07 & 1.59 \\
\hline $8 \times 8$ & 2.44 & 1.92 & 2.44 & 1.91 & - & - & 1.99 & 1.81 & 1.99 & 1.81 & 2.21 & 1.65 \\
\hline
\end{tabular}

Table $\mathrm{V}$ lists the power loss comparison of the maximum and average power loss experienced by a light signal travelling from one input port to an output port of these routers. It can be seen that GWOR has the lowest maximum power loss and also the lowest average power loss among the six router designs.

Theoretically speaking, no tuning circuit is needed for passive MRRs used in GWORs. While in practical applications, the resonance wavelength of fabricated MRRs may be shifted from desired values due to fabrication misalignment or ambient thermal variation [28]. To compensate for this inherent fabrication imperfectness of MRRs, different methods can be used, including post-fabrication trimming techniques, such as electron beam trimming [19], ultraviolet (UV) trimming [29], and doping the desired waveguide regions with $p$-type or $n$-type dopants [22]. As pointed out in [28], MRRs are extremely sensitive to temperature variation. A $1^{\circ} \mathrm{C}$ temperature change can cause the shift of the resonance wavelength by as much as $\sim 0.1$ $\mathrm{nm}$ [29]. The thermal sensitivity of MRRs can be reduced by proper design of waveguides and MRRs. In [29], a slotted MRR upper-clad with polymethyl methacrylate (PMMA, which has the opposite TO coefficient compared to the silicon material) has been introduced. The experimental results in [29] show that the temperature dependence of a PMMA-slotted MRR is only $27 \mathrm{pm} /{ }^{\circ} \mathrm{C}$ compared with $91 \mathrm{pm} /{ }^{\circ} \mathrm{C}$ for a regular MRR. It is shown by simulation in [29] that a zero thermal sensitivity condition can be achieved with a careful design in the future.

In practice, TO or EO effect-based ( $p-i-n$ diodes) tuning circuits are typically used to adjust the resonance shift caused by thermal variation. When TO tuning is employed, the power consumption caused by tuning is analyzed below. In GWORs, MRRs of different sizes are used and the channel spacing can be set large enough to avoid the overlap of adjacent channels under the maximum temperature variation. As such, for a given level of thermal variation, on each routing path of a GWOR, only those MRRs with the resonance wavelength corresponding to the input wavelength (based on the routing wavelength assignment) need to be tuned or detuned. For instance, in $4 \times 4$ GWOR (Fig. 2), the maximum and average numbers of MRRs that are needed to be tuned/detuned are two [for example, on path $I_{1} \rightarrow O_{3}$ in Fig. 2(a)/ and 4/3, respectively. Assuming the thermal tuning efficiency $0.91 \mathrm{~nm} / \mathrm{mW}$ as reported in [4], one can see that the total tuning power needed is very small.

\section{REDUNDANT GWOR STRUCTURE}

As shown in previous two sections, an $N \times N$ GWOR is capable of routing any combination of $N$ input-output pairs given $N-1$ input wavelengths. However, in this case, there is only

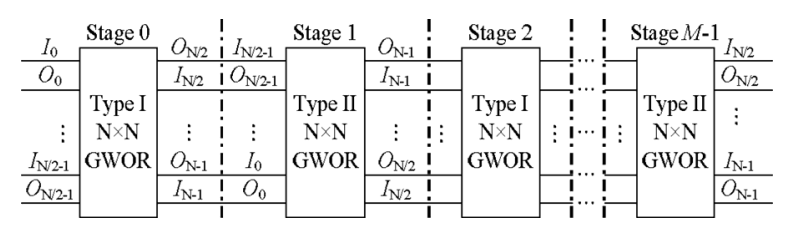

Fig. 6. Schematic of an $N \times N M$-RGWOR structure.

single routing path between any input-output pair. Based on the basic GWOR structures, in this section, we propose a redundant GWOR (RGWOR) structure so that a set of different wavelengths can be used to route data on multiple paths between any input-output pair.

To construct a RGWOR that provides $M(M \geq 2)$ routing paths between any indirectly connected source-destination pair, Type I and Type II GWORs are used as the basic building blocks (similarly, Type III and Type IV GWORs can be used when applicable). Fig. 6 shows the structure of an $N \times N$ RGWOR with $M$ routing paths between each indirectly connected input-output pair, and this structure is designated as $N \times N M$-RGWOR. The $N \times N M$-RGWOR is constructed by overlaying $M$ basic $N \times N$ GWORs; that is, there are $M$ different stages (referred as stages $0,1,2, \ldots$, and $M-1$ ) in $N \times N M$-RGWOR, among which at even stages are made of Type I $N \times N$ GWORs, while odd stages are made of Type II $N \times N$ GWORs. Alternatively, the $N \times N M$-RGWOR can also be built with Type II $N \times N$ GWORs at even stages and Type I $N \times N$ GWORs at odd stages.

As shown in Fig. 6, the $N$ pairs of ports in an $N \times N$ GWOR are evenly divided into two groups (Section IV). When $N=2 n$, the first group contains ports $\left(I_{0}, O_{0}\right),\left(I_{1}, O_{1}\right), \ldots,\left(I_{N / 2-1}, O_{N / 2-1}\right), \quad$ and the second group contains $\left(I_{N / 2}, O_{N / 2}\right),\left(I_{N / 2+1}, O_{N / 2+1}\right)$, $\ldots,\left(I_{N-1}, O_{N-1}\right)$; when $N=2 n+1$, the first group contains ports $\quad\left(I_{0}, O_{0}\right),\left(I_{1}, O_{1}\right), \ldots,\left(I_{(N-1) / 2-1}, O_{(N-1) / 2-1}\right)$ and $I_{(N-1) / 2}$, while the second group contains $O_{(N-1) / 2},\left(I_{(N+1) / 2}, O_{(N+1) / 2}\right),\left(I_{(N+1) / 2+1}, O_{(N+1) / 2+1}\right)$, $\ldots,\left(I_{N-1}, O_{N-1}\right)$. For each GWOR, at stage $k(0 \leq k \leq M-1)$ of the $N \times N M$-RGWOR, denote $I_{k, i}$ as the $i$ th input port and $O_{k, j}$ for the $j$ th output port $(0 \leq i \leq N-1,0 \leq j \leq N-1)$. The adjacent GWORs are connected according to the following rules:

1) For the GWOR at stage $k(0<k<M-1)$, connect its first port group to the second port group of the GWOR at stage $k-1$ using the waveguides, $I_{k, i} \rightarrow O_{k-1, j}$ and $O_{k, i} \rightarrow I_{k-1, j}(j=N-1-i)$, and connect its second port group to the first port group of the GWOR at stage $k+1$ using the waveguides, $I_{k, i} \rightarrow O_{k+1, j}$ and $O_{k, i} \rightarrow I_{k+1, j}$ (where $j=N-1-i$ ). No intersections of waveguides are allowed to form when connecting two adjacent GWORs.

2) For the GWOR at stage 0 , only connect its second port group to the first port group of the GWOR at stage 1. For the GWOR at stage $M-1$, only connect its first port group to the second port group of the GWOR at stage $M-2$ in the same manner as described in Rule 1).

Following the above connection rules, the waveguides connecting the adjacent GWORs will not intersect with other 


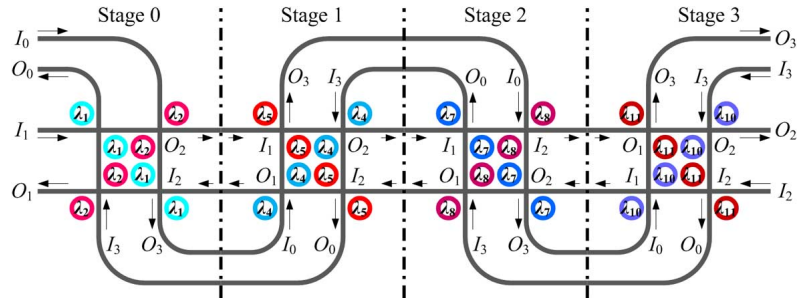

Fig. 7. Structure of the $4 \times 44-$ RGWOR.

TABLE VI

Routing WAVELENGTH ASSignMENT OF THE $4 \times 4$ 4-RGWORS

\begin{tabular}{|c|c|c|c|c|}
\hline & $O_{0}$ & $O_{1}$ & $O_{2}$ & $O_{3}$ \\
\hline$I_{0}$ & - & $\lambda_{1}, \lambda_{4}, \lambda_{7}, \lambda_{10}$ & $\lambda_{2}, \lambda_{5}, \lambda_{8}, \lambda_{11}$ & $\lambda_{3}, \lambda_{6}, \lambda_{9}, \lambda_{12}$ \\
\hline$I_{1}$ & $\lambda_{1}, \lambda_{4}, \lambda_{7}, \lambda_{10}$ & - & $\lambda_{3}, \lambda_{6}, \lambda_{9}, \lambda_{12}$ & $\lambda_{2}, \lambda_{5}, \lambda_{8}, \lambda_{11}$ \\
\hline$I_{2}$ & $\lambda_{2}, \lambda_{5}, \lambda_{8}, \lambda_{11}$ & $\lambda_{3}, \lambda_{6}, \lambda_{9}, \lambda_{12}$ & - & $\lambda_{1}, \lambda_{4}, \lambda_{7}, \lambda_{10}$ \\
\hline$I_{3}$ & $\lambda_{3}, \lambda_{6}, \lambda_{9}, \lambda_{12}$ & $\lambda_{2}, \lambda_{5}, \lambda_{8}, \lambda_{11}$ & $\lambda_{1}, \lambda_{4}, \lambda_{7}, \lambda_{10}$ & - \\
\hline
\end{tabular}

TABLE VII

ROUTING WAVELENGTH ASSIGNMENT OF THE $5 \times 5$ 4-RGWORS

\begin{tabular}{|c|c|c|c|c|c|}
\hline & $O_{0}$ & $O_{1}$ & $O_{2}$ & $O_{3}$ & $O_{4}$ \\
\hline$I_{0}$ & - & $\lambda_{1}, \lambda_{5}, \lambda_{9}, \lambda_{13}$ & $\lambda_{2}, \lambda_{6}, \lambda_{10}, \lambda_{14}$ & $\lambda_{3}, \lambda_{7}, \lambda_{11}, \lambda_{15}$ & $\lambda_{4}, \lambda_{8}, \lambda_{12}, \lambda_{16}$ \\
\hline$I_{1}$ & $\lambda_{4}, \lambda_{8}, \lambda_{12}, \lambda_{16}$ & - & $\lambda_{1}, \lambda_{5}, \lambda_{9}, \lambda_{13}$ & $\lambda_{2}, \lambda_{6}, \lambda_{10}, \lambda_{14}$ & $\lambda_{3}, \lambda_{7}, \lambda_{11}, \lambda_{15}$ \\
\hline$I_{2}$ & $\lambda_{3}, \lambda_{7}, \lambda_{11}, \lambda_{15}$ & $\lambda_{4}, \lambda_{8}, \lambda_{12}, \lambda_{16}$ & - & $\lambda_{1}, \lambda_{5}, \lambda_{9}, \lambda_{13}$ & $\lambda_{2}, \lambda_{6}, \lambda_{10}, \lambda_{14}$ \\
\hline$I_{3}$ & $\lambda_{2,}, \lambda_{6}, \lambda_{10}, \lambda_{14}$ & $\lambda_{3}, \lambda_{7}, \lambda_{11}, \lambda_{15}$ & $\lambda_{4}, \lambda_{8}, \lambda_{12,}, \lambda_{16}$ & - & $\lambda_{1}, \lambda_{5}, \lambda_{9}, \lambda_{13}$ \\
\hline$I_{4}$ & $\lambda_{1}, \lambda_{5}, \lambda_{9}, \lambda_{13}$ & $\lambda_{2}, \lambda_{6}, \lambda_{10}, \lambda_{14}$ & $\lambda_{3}, \lambda_{7}, \lambda_{11}, \lambda_{15}$ & $\lambda_{4}, \lambda_{8}, \lambda_{12}, \lambda_{16}$ & - \\
\hline
\end{tabular}

waveguides. This property can be seen from an example of a $4 \times 4$ 4-RGWOR shown in Fig. 7.

The wavelength assignment of a RGWOR is determined by three factors: the input port number, the output port number, and the stage that the GWOR belongs to. For an $N \times N M$-RGWOR, given the input-output pair $I_{i} \rightarrow O_{j}$, the input wavelength which shall make turn in the GWOR at stage $k(0 \leq k \leq M-1)$ is determined by

$$
C_{k}(i, j)=k(N-1)+C_{0}(i, j)
$$

where $k$ is the stage of the GWOR, and $C_{0}(i, j)$ is the input wavelength assigned to $N \times N$ GWOR given $I_{i} \rightarrow O_{j}$ (by (3) for $N$ is even or (4) for $N$ is odd). The wavelength assignments of the $4 \times 44$-RGWOR and $5 \times 54$-RGWOR are shown in Tables VI and VII, respectively.

Properties of an $N \times N M$-RGWOR are summarized below.

Lemma 5: For an $N \times N M$-RGWOR, given the input-output pair $I_{i} \rightarrow O_{j}$ (where $i \neq j$, and $i+j \neq N-1$ ), there exist $M$ routing paths when using $M$ different input wavelengths.

Proof: According to the construction rules of an $N \times N M$-RGWOR, there is no waveguide intersection between any two adjacent GWORs. As such, on the GWOR, at each stage of an $N \times N M$-RGWOR, each waveguide has one and only one intersection with each of the waveguide in other waveguide groups. Therefore, totally $M$ intersections are formed between one waveguide with any waveguide in other waveguide groups. At each of such intersection, the MRR corresponding to the input wavelength determined by (6) is assigned to a given input-output pair $I_{i} \rightarrow O_{j}$ (where $i \neq j$, and $i+j \neq N-1$ ). Totally, there are $M$ distinct MRRs assigned and each of these MRRs determines a routing path from $I_{i}$ to $O_{j}$. Note that the $M$ logical routing paths may share some waveguides. Hence, the Lemma holds.

Proposition 6: The $N \times N M$-RGWOR is non-blocking.

Proof: The basic $N \times N$ GWOR is non-blocking (Propositions 2 and 4). According to Lemma 5, from any input port of an $N \times N M$-RGWOR, all the other $(N-1)$ outputs are reachable with $M$ routing paths (assuming no self-communication is allowed). Following the wavelength assignment governed by (6), one can see that the light signals travelling between different input-output pairs through the same waveguide do not conflict with each other because these signals use different wavelengths. Hence the proposition holds.

Compared with GWOR, RGWOR can support higher bandwidth by having more than one input wavelength available for routing light signals between any single input-output pair. Another distinct advantage of RGWOR is its fault-tolerance capability by providing multiple routing paths between any inputoutput pair. When one of the routing path between an inputoutput pair fails due to the malfunction of the MRR, the remaining paths can still help maintain the connectivity between the input and output ports.

\section{CONCLUSION}

In this paper, we proposed a generic passive non-blocking router architecture named as GWOR, which can be scaled up from the basic $4 \times 4$ to any larger size. Routing in GWORs is realized by adopting different input wavelengths and MRRs with different geometries set by the pre-assigned wavelengths. The wavelength assignment schemes are derived for GWORs with both even and odd numbered input/output ports. In essence, an $N \times N$ GWOR needs $N-1$ input wavelengths, and $N(N-2)$ (for $N=2 n$ ) or $(N-1)^{2}$ (for $N=2 n+1$ ) MRRs for routing. Compared with the existing non-blocking router designs, GWOR uses the least number of MRRs and causes the lowest power loss for the light signal traveling from an input port to an output port. In addition, the passive nature of GWORs excludes the power needed to drive the TO/EO tuning and control circuits as needed by those active optical routers. As such, the proposed GWOR can serve as the building blocks for future high performance, power-efficient photonic NoCs. To provide multiple routing paths between any given input-output pair, we also proposed a redundant GWOR structure built by cascading basic GWORs at different stages.

\section{REFERENCES}

[1] S. Assefa, F. Xia, and Y. A. Vlasov, "Reinventing germanium avalanche photodetector for nanophotonic on-chip optical interconnects," Nature, vol. 464, pp. 80-84, Mar. 2010.

[2] C. Batten et al., "Building manycore processor-to-DRAM networks with monolithic silicon photonics," in Proc. 16th IEEE Symp. High Perform. Interconnects, Aug. 2008, pp. 21-30.

[3] M. Briere et al., "System level assessment of an optical NoC in an MPSoC platform," in Proc. Design, Auto. Test Eur. Opt. Conf. Exhib., 2007, pp. 1-6.

[4] P. Dong et al., "Low power and compact reconfigurable multiplexing devices based on silocon microring resonators," Opt. Exp., vol. 18, no. 10, pp. 9852-9858, Apr. 2010.

[5] A. W. Fang et al., "A racetrack mode-locked silicon evanescent laser," Opt. Exp., vol. 16, no. 2, pp. 1393-1398, Jan. 2008. 
[6] H. Gu, K. H. Mo, J. Xu, and W. Zhang, "A low-power low-cost optical router for optical networks-on-chip in multiprocessor systems-onchip," in Proc. IEEE Comp. Soc. Annu. Symp. VLSI (ISVLSI), 2009, pp. $19-24$.

[7] A. Huang et al., "A $10 \mathrm{~Gb} / \mathrm{s}$ photonic modulator and WDM MUX/ DEMUX integrated with electronics in $0.13 / \mathrm{spl} \mathrm{mu} / \mathrm{m}$ SOI CMOS," in Proc. IEEE Int. Solid-State Circuits Conf. (ISSCC), 2006, pp. 922-929.

[8] "The international technology roadmap for semiconductors (ITRS)," 2009 [Online]. Available: http://www.itrs.net/Links/2009ITRS/ 2009Chapters 2009Tables/2009 Interconnect.pdf

[9] N. Kirman and J. F. Martinez, "A power-efficient all-optical on-chip interconnect using wavelength-based oblivious routing," $A C M$ SIGARCH Comp. Architect. News, vol. 38, no. 1, pp. 15-28, Mar. 2010.

[10] M. Lipson, "Guiding, modulating, and emitting light on silicon-challenges and opportunities," J. Lightw. Technol., vol. 23, no. 12, pp. 4222-4238, Dec. 2005.

[11] M. Lipson, "Compact electro-optic modulators on a silicon chip," IEEE J. Sel. Topics Quantum Electron., vol. 12, no. 6, pp. 1520-1526, Nov. 2006.

[12] B. E. Little, S. T. Chu, W. Pan, and Y. Kokubun, "Microring resonator arrays for VLSI photonics," IEEE Photon. Technol. Lett., vol. 12, no. 3, pp. 323-325, Mar. 2000.

[13] A. Liu, "Announcing the world's first 40G silicon laser modulator," Intel, 2007 [Online]. Available: http://blogs.intel.com/research-/2007/07/40g_modulator.php

[14] R. Morrisand and A. K. Kodi, "Exploring the design of 64- and 256core power efficient nanophotonic interconnect," IEEE J. Sel. Topics Quantum Electron., vol. 16, no. 5, pp. 1386-1393, Oct. 2010.

[15] H. Park et al., "A hybrid AlGaInAs-silicon evanescent waveguide photodetector," Opt. Exp., vol. 15, no. 10, pp. 6044-6052, May 2007.

[16] A. W. Poon, F. Xu, and X. Luo, "Cascaded active silicon microresonator array cross-connect circuits for WDM networks-on-chip," in Proc. SPIE Int. Soc. Opt. Eng., 2008, vol. 6898, pp. 12-21.

[17] A. W. Poon, F. Xu, and X. Luo, "Cascaded microresonator-based matrix switch for silicon on-chip optical interconnect," Proc. IEEE, vol. 97, no. 7, pp. 1216-1238, Jul. 2009.

[18] L. Schares et al., "A 17-Gb/s low-power optical receiver using a Ge-on-SOI photodiode with a $0.13-\mu \mathrm{m}$ CMOS IC," in Proc. Opt. Fiber Commun. Conf. (OFC), 2006, pp. 1-3.

[19] J. Schrauwen, D. V. Thourhout, and R. Baets, "Trimming of silicon ring resonaror by electron beam induced compaction and strain," Opt. Exp., vol. 16, no. 6, pp. 3738-3743, Mar. 2008.

[20] A. Shacham, K. Bergman, and L. P. Carloni, "Photonic networks-on-chip for future generations of chip multiprocessors," IEEE Trans. Comput., vol. 57, no. 9, pp. 1246-1260, Sep. 2008.

[21] N. Sherwood-Droz et al., "Optical $4 \times 4$ hitless silicon router for optical networks-on-chip (NoC)," Opt. Exp., vol. 16, no. 20, pp. 15915-15922, Sep. 2008

[22] R. Soref and B. Bennett, "Electrooptical effects in silicon," IEEE $J$. Quantum Electron., vol. 23, no. 1, pp. 123-129, Jan. 1987.

[23] Y. Tao et al., " $40 \mathrm{~Gb} / \mathrm{s}$ Ge-on-SOI waveguide photodetectors by selective Ge growth," in Proc. Opt. Fiber Commun./Nat. Fiber Opt. Eng. Conf., 2008, pp. 1-3.

[24] F. Xia, M. Rooks, L. Sekaric, and Y. Vlasov, "Ultra-compact high order ring resonator filters using submicron silicon photonic wires for on-chip optical interconnects," Opt. Exp., vol. 15, no. 19, pp. 11934-11941, Sep. 2007.

[25] Q. Xu, B. Schmidt, S. Pradhan, and M. Lipson, "Micrometre-scale silicon electro-optic modulator," Nature, vol. 435, no. 7040, pp. 325-327, May 2005.
[26] L. Zhang, M. Yang, Y. Jiang, E. Regentova, and E. Lu, "Generalized wavelength routed optical micronetwork in network-on-chip," in Proc. 18th IASTED Int. Conf. Parallel Dist. Comp. Syst., 2006, pp. 698-703.

[27] L. Zhang, M. Yang, Y. Jiang, and E. Regentova, "Architectures and routing schemes for optical network-on-chips," Comp. Elec. Eng., vol. 35, no. 6, pp. 856-877, Nov. 2009.

[28] L. Zhou, S. S. Djordjevic, R. Proietti, D. Ding, S. J. B. Yoo, R. Amirtharajah, and V. Akella, "Design and evaluation of an arbitration-free passive optical crossbar for on-chip interconnection networks," Appl. Phys. A., vol. 95, pp. 1111-1118, Feb. 2009.

[29] L. Zhou, K. Okamoto, and S. J. B. Yoo, "Athermalizing and trimming of slotted silicon microring resonators with UV-sensitive PMMA upper-cladding," IEEE Photon. Technol. Lett., vol. 21, no. 17, pp. $1175-1177$, Feb. 2009.

Xianfang Tan received the M.S. degree in electrical engineering from Tianjin University, Tianjin, China, and the M.S. degree in mechanical engineering from the University of Nevada, Las Vegas (UNLV) in 2003 and 2005, respectively, where she is currently working toward the Ph.D. degree in electrical engineering.

Her current research interests include computer architectures, on-chip interconnection networks and VLSI architectures.

Mei Yang received the Ph.D. degree in computer science from the University of Texas, Dallas, in 2003.

She is currently an Associate Professor in the Department of Electrical and Computer Engineering, University of Nevada, Las Vegas. Her research interests include computer architectures, on-chip interconnection networks, embedded systems, and networking.

Lei Zhang received the Ph.D. degree in electrical engineering from the University of Nevada, Las Vegas in 2011.

His research interests include computer architectures, on-chip interconnection networks, optoelectronics and image processing.

Yingtao Jiang received the $\mathrm{Ph}$.D. degree in computer science from the University of Texas, Dallas, in 2001 .

He is currently an Associate Professor in the Department of Electrical and Computer Engineering, University of Nevada, Las Vegas. His current research interests include algorithms, VLSI architectures, and circuit level techniques for the design of DSP, computer architectures, nanotechnologies, sensors and instrumentation.

Jianyi Yang received the B.S., M.S., and Ph.D. degrees in electronic science and technology from Zhejiang University, Hangzhou, China, in 1991, 1994, and 1996, respectively.

$\mathrm{He}$ is currently a Professor in the Department of Information Science and Electronics Engineering, Zhejiang University. His interests include integrated optics and silicon photonics, optical interconnects, and optical sensors. 